\title{
Maternal Nutrition and Epigenetic Perturbation: Modeling Trends to Translation
}

\author{
Judy L. Oakes $\cdot$ Folami Y. Ideraabdullah
}

Published online: 24 July 2013

(c) Springer Science + Business Media New York 2013

\begin{abstract}
Maternal nutrition plays an essential role in offspring health and development. Critical stages include: (1) preconception, affecting oocyte development and uterine environment preparation; (2) gestation, affecting uterine environment and placental nutrient transfer and (3) postnatal, through lactation. It remains debatable which stage is most important, but arguably, the most complex cellular events occur during gestation. During this time, embryo development requires a well orchestrated and tightly regulated cascade of genetic, molecular and biochemical events. Among these are epigenetic events necessary for gene expression regulation. These produce heritable yet often reversible states established based on transcriptional needs of the cell. A growing body of research highlights the role of maternal nutrition in determining epigenetic states important for pre- and postnatal development. Here, we discuss recent findings addressing epigenetic response to nutrition with relevance to developmental origins of phenotypic outcome.
\end{abstract}

Keywords Nutrition - Maternal nutrition .

Developmental origins of adult disease $\cdot$ Fetal origins of adult disease - Epigenetics - Epigenetic inheritance . Nutrition and heritability - Aggregate effects - Nutrient interactions - Nutrient-toxicant interactions - Nutrientgene interactions · Gene-environment interactions - Fetal development - Disease origins - Windows of susceptibility

J. L. Oakes · F. Y. Ideraabdullah ( $\bowtie)$

University of North Carolina at Chapel Hill Nutrition Research Institute, 500 Laureate Way, Kannapolis, NC 28081, USA

e-mail: folami_ideraabdullah@unc.edu

F. Y. Ideraabdullah

Department of Genetics, University of North Carolina at Chapel Hill, Chapel Hill, NC 27599, USA

\section{Introduction}

Developmental Origins of Disease

The concept of fetal origins of adult disease is a paradigm that started with the "Barker's Hypothesis" [1]. The concept was initially met with resistance, as studies could not definitively limit disease origins to fetal stages and the presence of numerous other confounding factors in human studies limited the ability to fully test the hypothesis [2]. The more broadly encompassing concept of developmental origins of adult disease (DOAD), which implicates the role of development before, during and after gestation in later stages of health and fitness, is more widely accepted but also remains difficult to support scientifically. This is in part due to the fact that while there is a seemingly clear correlation between these early developmental stages and phenotypic outcome later in life, disease mechanisms and modes of action remain elusive. Here, we discuss recent findings supporting the role of maternal nutrition in developmental origins of disease as it pertains specifically to epigenetic mechanisms. We examine new molecular mechanisms and discuss potential difficulties in dissecting these findings that may delay production of efficient preventative or treatment measures.

The Role of Epigenetic Mechanisms in DOAD

Epigenetic regulation is an essential form of gene expression regulation. A wide variety of specific epigenetic regulatory factors have been identified and are relatively well-characterized including DNA methylation and hydroxymethylation, post-translational modifications to histones, and non-coding RNA. These epigenetic 
mechanisms often work together and are associated with gene activation or repression depending on the resulting chromatin structure, the type of modification, the genomic location of the modification and the timing or cellular location of epigenetic events [3, 4]. Of utmost importance in the link between nutrition and epigenetic mechanisms is the flexibility of epigenetic states that is not present with genetic (DNA sequence) states. Like DNA sequence, epigenetic states can be heritable. States at some loci are heritable from parent to child (e.g. imprinted loci) while states at many loci are only "heritable" from cell to cell through replication. Unlike most DNA sequences, many epigenetic states can be changed. These changes are a necessary part of normal cellular function (e.g. promoter methylation changes during cell differentiation) but can also occur in response to exogenous perturbation. A growing number of both essential and non-essential nutrients perturb epigenetic states. Of particular interest are nutrients that perturb epigenetic states programmed during early development that contribute to offspring health and fitness outcomes. These include members of the one-carbon metabolism pathway such as folate, choline and zinc, fatty acids, proteins and essential vitamins A and D. Recent studies have elucidated new mechanisms, new roles in disease, new susceptibility factors including timing of nutrient intake and genetic influences, and a new understanding of aggregate interactions.

In support of the concept of DOAD, many diseases have been linked to maternal nutrition and epigenetic states in the offspring. These include, but are not limited to, diseases directly associated with nutrient intake such as non-alcoholic fatty liver disease [5-7] developmental defects such as delay or disruption of embryo or organ development [812], fertility defects [13, 14], neurological disorders [15], and complex traits such as obesity and diabetes [16-19]. Roles in inflammation and cancer have also been reported [20-25]. Here, we focus on recently elucidated molecular mechanisms of nutrient involvement in determining epigenetic signatures during early development. These include studies determining windows of susceptibility to nutrientinduced epigenetic response, direct and indirect effects and mechanisms of heritability. We also discuss the role of aggregate effects between maternal nutrition and other environmental stimuli such as other nutrients, genomic environment, and toxicant/drug interactions. Such interactions may clarify some of the unexplained diversity in phenotypic response to nutrient intake and also potentially some of the discord between experiments. These recent findings illuminate our understanding of developmental origins of disease but also show limitations and areas needing more stringent control when translating results to human populations.

\section{Molecular Mechanisms}

Determining molecular mechanisms of nutritional influence on epigenetic states during early development starts with determining windows of greatest susceptibility. However, determining accurate windows of susceptibility requires an understanding of the complex system of epigenetic mechanisms and timing of events. One could argue that potential epigenetic dysregulation resulting from nutritional changes would likely occur within a time period where the greatest number of epigenetic changes occurs in the shortest amount of time. Embryogenesis certainly meets that criterion such that immediately following fertilization the embryo begins setting up the many different epigenetic profiles required for normal cellular processes in different cell types. These elegantly orchestrated epigenetic events are required for normal development of a multifunctioning, multicellular organism. Dysregulation of events that establish these required epigenetic profiles may perturb normal development during embryogenesis or perinatal development with the potential for late onset effects through adulthood. While scientific evidence on the interplay of epigenetics and nutrients builds, few have as yet, identified direct mechanisms involved. This section will briefly review recently examined mechanisms involved in nutrient-induced epigenetic changes within susceptible developmental time points and discuss modes of heritability.

Windows of Susceptibility

Potential windows of susceptibility where maternal nutrition may influence offspring epigenome include pre-conception, during gestation and postnatal during lactation. During these stages, epigenetic states within a cell change depending on current or future transcriptional needs. Genome-wide, DNA methylation is erased and reset in a process known as epigenetic reprogramming [26]. This occurs once during germ cell development (partly occurring in utero) and again immediately after fertilization. This reprogramming serves two purposes: (1) it prepares the cell for a new fate allowing the necessary genes to be activated or repressed, and (2) it erases previously acquired defects allowing for a new start with each generation. In addition to these genome-wide events, throughout embryonic and postnatal development, epigenetic states at specific genes or groups of genes within a pathway are programmed to meet the current or future needs of the cell. Because epigenetic states are determined based on timing and cell type, windows of susceptibility will depend on the phenotypic effect being investigated. Exciting new research has only begun to investigate how nutrientinduced epigenetic effects early in development may contribute to phenotypes in adulthood, during aging or those 
transmitted through multiple generations. Here, we will discuss a few recent findings that elucidate the timing of susceptibility windows during early development.

\section{Preconception}

Maternal nutrition before conception can affect epigenetic states in the oocyte, which may affect both oocyte development and postfertilization potential of the embryo. Recently, mouse oocyte quality was shown to be affected by maternal zinc deficiency during a 3-5 day window before ovulation [27]. According to their study, zinc deficiency during this time resulted in a significant decrease in global trimethylation of histone 3 Lysine 4 (H3K4me3, associated with gene activation) and global DNA methylation in superovulated GV oocytes [27]. These epigenetic defects were associated with fertilization potential, preimplantation embryo development and transcriptional perturbation at repeat elements and genes essential to embryo development. Zinc is an essential nutrient known to play a role in biosynthesis of $S$-adenosylmethionine (SAM), the substrate required for methyl group transfer [28]. Tian and Diaz [27] also showed that SAM supplementation rescues the effects of pre-ovulatory zinc deficiency on H3K4me3 and fertility potential. Studies such as this demonstrate that even very narrow windows of nutrient fluctuations may have significant repercussions on epigenetic states depending on timing and likely also the nutrient involved.

\section{Gestation}

Mouse models show that maternal choline deficiency between embryonic day 12 (E12) and E17 results in decreased global and gene-specific ( $C d k n 3$, cyclin-dependent kinase inhibitor 3) DNA methylation in the E17 fetal brain (ventricular and sub-ventricular zones of Ammon's horn) [29]. DNA methylation changes at $C d k n 3$ (a cell proliferation inhibitor) was correlated with protein levels and is consistent with previous findings of reduced cell proliferation within the Ammon's horn associated with choline deficiency $[30,31]$. In a separate study using rat models, choline availability through enrichment or deficiency between E11 and E17 was linked to DNA methylation changes at histone methyltransferase genes, $G 9 a$ and Suv39hl in E17 liver [32]. These changes were correlated with RNA and protein expression levels at these loci as well as levels of active (H3K4me2) and repressive (H3K27me3 and H3K9me2) histone modifications.

\section{Postnatal}

The least investigated effects are those occurring after birth during lactation. Recently Niculescu et al. [33•] investigated the effect of perturbation of $\alpha$-linolenic acid (ALA) intake on DNA methylation and $\omega-3 / \omega-6$ fatty acid metabolism pathways. Using mouse models, they found epigenetic defects present in offspring (liver) from dams that were ALA deficient before, during and after gestation, were absent in offspring from dams that were fed ALA diets before and during gestation but supplemented with ALA during lactation. This important finding demonstrates that epigenetic effects induced by nutrient intake during preconception and/or gestation may be rescued by postnatal dietary changes. It also brings to light the concept that windows of rescue may exist for epigenetic defects incurred in the fetus.

\section{Direct and Indirect Effects}

In addition to difficulties pinpointing windows of susceptibility, another major difficulty in dissecting roles of nutrition in epigenetic states during development is the inability to identify direct mechanisms of perturbation. For many of the epigenetic defects and downstream phenotypes it remains unclear whether the link to nutrition is directly linked to nutrient pathways or indirect effects of global dysregulation and whether the epigenetic effects observed are causal or the result of other unrelated defects.

Many recent studies target the methyl donor pathway to identify mechanisms of nutrient-induced epigenetic perturbation. Methyl donor nutrients are part of the more complex one-carbon metabolism pathway that involves carrying, transfer and activation of carbon units required for serine to glycine conversion, purine and pyrimidine synthesis required for DNA and RNA synthesis and repair, and methyl group transfer required for DNA methylation [34]. The latter activity highlights a potential direct link between methyl donor nutrients (folate, choline, betaine, vitamin $B_{12}$, and zinc) and epigenetic states during development. This initially suggests a simplified view of negative effects of deficiency and positive effects of supplementation. However, new findings elucidate the complexity of effects induced by altering methyl donor nutrient intake such that both deficiency and enrichment results in epigenetic perturbation creating a u-shaped response curve. Furthermore, interactions between nutrients in the methyl donor pathway and the potential involvement of indirect effects caused by other non-DNA methylation related functions of the one-carbon metabolism pathway cannot be ignored when extrapolating results.

Aside from the methyl donor pathway, additional mechanisms modulate epigenetic states. Vitamin A deficiency prior to and during gestation was shown to be associated with abnormal heart development and altered promoter methylation and expression of the transcription factor Gata-4 in the heart [35]. These defects coincided 
with significant expression changes at DNA methyltransferase genes (Dnmt): increase in Dnmtl and decrease in $D n m t 3 a$ and Dnmt $3 b$. Interestingly, vitamin A supplementation partway through gestation restored Gata-4 promoter methylation but did not completely restore Gata-4 mRNA levels. These findings link vitamin A to DNA methylation through DNMT function as a potential source of methylation perturbation.

In a potentially unrelated mechanism, low-protein diets in porcine models during the gestational-lactation period were shown to alter offspring body and organ weight [36]. These phenotypic changes were associated with serum and liver cholesterol levels as well as hepatic upregulation of genes involved in cholesterol biosynthesis and metabolism (3-hydroxy-3-methylglutaryl-CoA, HMGCR and cholesterol-7-alpha-hydroxylase, CYP7 $\alpha 1$ ). Gene expression changes were attributed to decreased trimethylation of histone 3 Lysine 27 (H3K27me3, a repressive histone mark). A separate study showed that both dietary protein enrichment and deficiency resulted in significant changes in expression of Dnmt1, Dnmt3a and Dnmt3b and hepatic global methylation [37]. Interestingly, the severity of effects on global DNA methylation was observed at different stages of embryonic development (dpc95 and dpc188, respectively) depending on the diet (enriched or deficient), suggesting mechanistic differences.

Finally, vitamin D levels have also been shown to determine epigenetic states during fetal development. Active vitamin $\mathrm{D}$ metabolite, $1 \alpha, 25$-dihydroxyvitamin D3 $[1,25(\mathrm{OH}) 2 \mathrm{D} 3]$ was shown to induce expression of multiple histone demethylases including lysine-specific demethylases, $L S D 1$ and $L S D 2$, and members of the Jumonji C (JmjC) domain-containing demethylases including JMJD3, HSPBAP1, JARID2 and KDM5B. Meanwhile, 1,25(OH)2D3 inhibits expression of JmjC domain-containing demethylases KDM4A/4C/4D/5A/2B, HIFlAN, JMJD5/6, HR and $P L A 2 G 4 B[38 \cdot, 39]$. Histone methylation plays an important role in gene activation and repression depending on the histone residue that is methylated and whether it is mono-, dior tri- methylated. Although carried out in cell lines, these findings have significant implications for the role of nutrition in epigenetic states during early development and it is likely that ongoing studies will address this topic.

\section{Heritability}

Potential mechanisms of heritability may play a significant role in epigenetic defects acquired during early development such that heritable factors transmitted from parent to child may affect susceptibility to nutrient-induced epigenetic perturbation. Such mechanisms of heritability are poorly understood but here we will discuss a few recent studies that illustrate potentially relevant mechanisms.
Based on previous studies in humans, inheritance of naturally occurring genetic variants that alter function of nutrient metabolism genes (e.g. Mthfr, methylenetetrahydrofolate reductase), confers susceptibility to epigenetic defects [40-44]. It is hypothesized that these effects are due to altered biosynthesis of SAM through the methyl donor pathway.

A second potential mode of inheritance is through nutrient-induced DNA damage, particularly if the damage occurs during critical time points of embryogenesis or PGC development, are not properly (genetically and epigenetically) repaired, and do not eliminate function of essential genes. Deficiency in a combination of choline, folate and methionine in adult rat models resulted in accumulation of 8-oxodeoxyguanosine and 3_OH-end strand breaks in hepatic DNA [45]. A more recent study combining in utero folate depletion and postnatal high-fat diet reports marginally increased oxidative damage in the brain compared to in utero folate depletion alone; suggesting that DNA damage in the fetus creates increased susceptibility to postnatal effects of a high fat diet [46॰]. More importantly, the increase in oxidative DNA damage was associated with decreased base excision repair (BER), an activity previously linked to disruption of DNA methylation. BER components are upregulated in PGC's [47], thereby establishing a potential mechanistic link in timing between DNA repair and DNA methylation. Investigating the role of nutrition on such effects during germ cell development in the fetus will allow for identification of nutrientdependent effects with heritable consequences.

\section{Aggregate Interactions}

Aggregate interactions likely play a significant role in determining health and fitness outcomes related to nutrient availability during early development. These interactions are usually complex in nature and may explain why an individual is responsive or non-responsive to nutritional stimuli. Here, we discuss new findings regarding interactions with maternal nutrient intake that determine offspring epigenetic programming and ultimately offspring phenotypic outcomes. These include nutrient-nutrient, nutrient-gene, and nutrient-toxicant interactions. While the interactions discussed here are not all-inclusive and often are not examined in developmental models, they represent likely suspects for perturbing epigenetic states during early development and open new doors to understanding determinants of epigenetic fate.

\section{Nutrient-Nutrient}

Most nutrients work together in complex interacting pathways for proper use in cellular processes. Recent studies 
highlight the benefits of a balanced ratio of these nutrients with others, somewhat contradicting the benefits of supplementation of individual components.

Recent studies focus on interactions between nutrients that modulate fatty acid metabolism and bioavailability. The ratio of folate to vitamin $B_{12}$ levels was found to be essential for normal DNA methylation states in placenta [48]. While vitamin $B_{12}$ deficiency alone did not affect DNA methylation in placenta, the combination of $B_{12}$ deficiency and excess folate resulted in reduced global DNA methylation in placenta and lowered plasma and placental levels of the $\omega-3$ fatty acid, docosahexaenoic acid (DHA). These effects were rescued by supplementation with $\omega-3$ fatty acids, demonstrating a link between the two nutrient pathways. In support of a link between fatty acid pathways and 1-carbon metabolism, methyl donor nutrient supplementation (choline, betaine, vitamin B12, and folic acid) was shown to rescue effects of obesogenic high fatsucrose diet on hepatic triglyceride accumulation but exacerbated the effects on DNA methylation at the fatty acid synthase (Fasn) promoter and Fasn expression [49•]. Likewise, studies show that while high fat diet alone is sufficient to increase atherosclerotic plaque formation, folic acid deficiency exacerbates this effect [50]. Although these effects are correlated with SAM levels, global DNA methylation in affected tissues was not significantly altered. In the reciprocal study, epigenetic defects detected in offspring exposed to high fat diet during gestation and lactation were rescued with methyl donor nutrient supplementation (choline, betaine, folic acid, vitamin $\mathrm{B}_{12}$, and zinc) [51]. These studies demonstrate clear links between methyl donor nutrient pathways and fatty acid metabolism during early development and may provide clues to early origins of metabolic syndrome related profiles later in life.

Repeat exposure to nutrient enrichment was also recently investigated by analysis of epigenetic and developmental effects of three generations of maternal obesity in a mouse model [25]. Multigenerational exposure to high fat diet resulted in multigenerational effects on promoter methylation and expression at inflammatory response genes, Toll-like receptor genes 1 and $2(T l r 1, T l r 2)$ and the linker for activated T-cells (Lat). Interestingly, severity of epigenetic defects increased with each generation and was linked to Dnmtl expression changes. These studies highlight epigenetic response to repeat exposure to an obesogenic environment, a model that has direct relevance to human populations.

\section{Nutrient-Gene}

It is well acknowledged that genetic factors influence nutrient-induced effects. However, these interactions and how they affect epigenetic mechanisms are still poorly understood. Properly addressing this question requires thorough cross-disciplinary studies of genetics, epigenetics and nutrition and exploration of these interactions at the whole genome level, which remains a formidable but not impossible task. Many recent studies instead target specific loci.

DNA sequence changes at metabolic genes involved in the methyl donor pathways are most well studied with regards to nutrient-gene interactions [52]. When animals with dysfunctional alleles at these genes are challenged with methyl donor nutrient enrichment or deficiency, epigenetic states are seemingly perturbed further and correspond with an increase in adverse phenotypic outcome [53]. Likewise, humans carrying polymorphic alleles at these genes have increased risk of methylation defects [40].

Studies also address interactions between nutrition and disease risk alleles. Ortega-Azorin et al. [54] report a link between diet and methylation regulated obesity risk alleles at FTO and MC4R (Fat mass and obesity and melanocortin4 receptor, respectively) in determining type 2 diabetes risk. Alternatively, the role of as yet unidentified loci has been investigated. Choline and folate deficiency were shown to result in mouse strain dependent effects on expression at several one-carbon metabolism genes [55], lipid metabolism genes, and markers of oxidative stress [56]. While the effect on one-carbon metabolism genes implies potential downstream DNA methylation defects and strain dependent effects strongly indicate the involvement of genetic factors, specific epigenetic effects and genetic elements were not investigated. Follow-up studies will likely address these areas and provide additional clues as to how genetic variation regulates epigenetic response to nutrition.

\section{Nutrient-Toxicant}

Initial studies reported that maternal nutrient supplementation (methyl donor nutrients and genestein, a soy-based xenoestrogen) rescues offspring epigenetic defects at the Agouti locus caused by the endocrine disruptor bisphenol A (BPA) [57]. However, a separate study showed that maternal supplementation of genestein or BPA alone versus genestein and BPA combined surprisingly did not result in visible phenotypes of epigenetic defects at the Agouti locus [58]. Therefore, while nutrient supplementation presents a very appealing resolution to address toxicant exposure, these interactions are likely more complex than initially thought. Interestingly BPA and genestein have also been used to investigate additive roles of environmental compounds that mimic endogenous estrogens in disease. Diets containing both BPA and genestein induced more severe effects compared to either alone including phenotypic effects on the central nervous system [59] and 
molecular effects on estrogen receptor 1 (ESR1) binding, global gene expression and protein regulation [60]. DNA methylation changes were not explored but were implied. Conflicting results between these and earlier studies may have more to do with the variable nature of epigenetic perturbation by BPA rather than with rescue potential of nutrient supplementation, however both pathways require further investigation to clarify.

Additive interactions between other prevalent toxicants and nutrient intake include a recent study showing aggregate effects of prenatal high folate diet and arsenic exposure on mouse fetuses [61•]. Inorganic arsenic alone caused significant changes in body weight and SAM and SAH levels in fetal liver, an effect exacerbated by folate supplementation including additional $\mathrm{CpG}$ island methylation changes in fetal liver at about 2,931 genes. Such findings suggest that nutrient supplementation in some situations may be harmful when exposure to toxicants are present.

Finally, sex-specific effects introduce an additional level of complexity to aggregate nutrient interactions that has yet to be fully explored. Nohara et al. [62] report that combined methyl donor nutrient deficiency (methionine, folic acid and choline) and arsenic exposure reduces global DNA methylation levels in male mouse liver but increased global DNA methylation in female mouse liver. These findings require additional studies to determine the mechanism but have great implications of a role for sex-specific differences in nutrition-induced epigenetic changes.

\section{Conclusions}

As discussed above, many recent breakthroughs elucidate our understanding of the role of nutrition in epigenetic perturbation and disease outcome. However, these findings seemingly open a Pandora's box of limitless possibilities involving combinations of mechanisms, timings, and interactive effects that can perturb epigenetic states during early development and result in adverse phenotypic outcomes (Fig. 1). While many studies identify effects of nutrition on the epigenome during embryonic development, many ongoing studies aim to dissect mechanisms of perturbation. These will determine how multiple molecular pathways intersect to exacerbate or negate detrimental effects and whether these studies elucidate relevant solutions for human populations. Unfortunately, in many animal models, deficiencies or supplementations far exceed that found in human populations and results may represent the exaggerated effects of an overwhelmed biological system that may or may not occur in a human population. Also, the u-shaped dosage response of many nutrients such that dietary excess or deficiency has similar effects on the epigenome complicates efforts to understand mechanisms

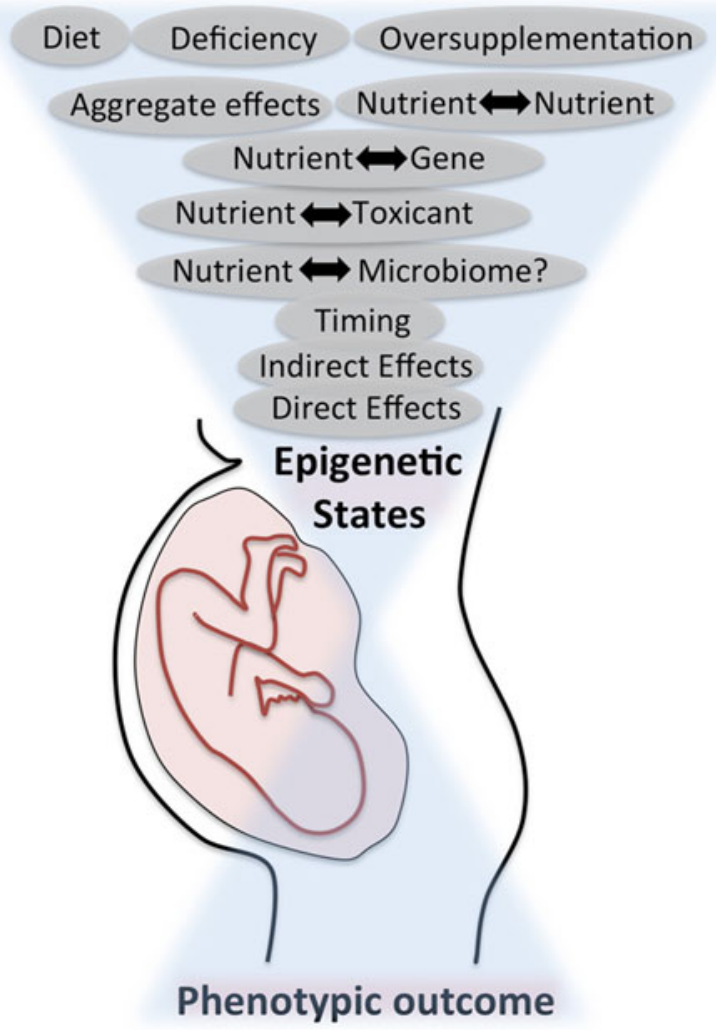

Fig. 1 Maternal nutrient influences on epigenetic programming during development

of perturbation. Overall, additional systematic and thorough studies are needed to support initial findings using animal models with human counterparts in mind for later translation.

Cross-disciplinary studies between genetics, epigenetics, nutrition and toxicology increase scientific capacity to dissect interacting pathways and aggregate effects. However, the results can be at times difficult to interpret. The development of new computational tools to analyze complex data and correlative relationships is required particularly in the area of epigenetic effects where statistical analysis still poses an especially difficult task. Of equal importance, computational tools and theoretical models are being developed to account for epigenetic variation in genetic association studies [63, 64]. Hopefully, this will increase power to identify previously inaccessible heritable disease markers.

Human studies represent a direct medium for addressing questions related to maternal nutrition and offspring outcome. However, limited access to epigenetically relevant tissues or cell types and control over timing of nutrient intake often prohibits identification of causal mechanisms and pathways. Increased interactions between researchers working with animal models and researchers with access to 
human phenotypic data and/or samples are helping alleviate these issues. In addition, support for studies using nontraditional model organisms (i.e. non-rodent) to answer questions related to maternal nutrition and offspring epigenetic perturbation may provide novel mechanistic links.

Finally, recent findings emphasize potentially adverse effects of nutrient supplementation and demonstrate the need for more studies examining aggregate effects as the basis of individual response. Such interactions will likely explain some of the contradicting findings between studies. Additional research areas needing expansion in order to fully understand the role of maternal nutrition in epigenetic origins of disease in the offspring include studies investigating the role of interactions between nutrition and the microbiome and the role of paternal nutrition. Both of these areas remain poorly developed but hopefully will be elucidated soon.

Acknowledgments We would like to thank Dr. Mihai Niculescu for reading this manuscript and providing comments.

Disclosure Judy L. Oakes and Folami Y. Ideraabdullah declare that they have no conflict of interest.

Human and Animal Rights and Informed Consent This article does not contain any studies with human or animal subjects performed by any of the authors.

\section{References}

Papers of particular interest, published recently, have been highlighted as:

- Of importance

1. Barker DJ. The fetal and infant origins of adult disease. BMJ. 1990;17(301):1111.

2. Paneth N, Susser M. Early origin of coronary heart disease (the "Barker hypothesis"). BMJ. 1995;310:411.

3. Bonasio R, Tu S, Reinberg D. Molecular signals of epigenetic states. Science. 2010;330:612-6.

4. Holliday R, Pugh JE. DNA modification mechanisms and gene activity during development. Science. 1975;187:226-32.

5. Teng YW, Mehedint MG, Garrow TA, Zeisel SH. Deletion of betaine-homocysteine $S$-methyltransferase in mice perturbs choline and 1-carbon metabolism resulting liver and hepatocellular carcinomas. J Biol Chem. 2011;4:1-10.

6. Mehedint MG, Zeisel SH. Choline's role in maintaining liver function: new evidence for epigenetic mechanisms. Curr Opin Clin Nutr Metab Care. 2013;16:339-45.

7. Corbin KD, Zeisel SH. Choline metabolism provides novel insights into nonalcoholic fatty liver disease and its progression. Curr Opin Gastroenterol. 2012;28:159-65.

8. Czeizel AEA, Dudás II. Prevention of the first occurrence of neural-tube defects by periconceptional vitamin supplementation. N Engl J Med. 1992;327:1832-5.

9. van Beynum IM, Kapusta L, Bakker MK, et al. Protective effect of periconceptional folic acid supplements on the risk of congenital heart defects: a registry-based case-control study in the northern Netherlands. Eur Heart J. 2010;31:464-71.

10. Czeizel AEA, Dudás II, Paput LL, Bánhidy FF. Prevention of neural-tube defects with periconceptional folic acid, methylfolate, or multivitamins? Ann Nutr Metab. 2011;58:263-71.

11. Christensen KE, Rohlicek CV, Andelfinger GU, et al. The MTHFD1 p.Arg653Gln variant alters enzyme function and increases risk for congenital heart defects. Hum Mutat. 2009;30:212-20.

12. Beaudin AE, Perry CA, Stabler SP, Allen RH, Stover PJ. Maternal Mthfd1 disruption impairs fetal growth but does not cause neural tube defects in mice. Am J Clin Nutr. 2012;95:882-91.

13. Johnson AR, Craciunescu CN, Guo Z, et al. Deletion of murine choline dehydrogenase results in diminished sperm motility. FASEB J. 2010;24:2752-61.

14. Forges T, Monnier-Barbarino P, Alberto JM, Gueant-Rodriguez RM, Daval JL, Gueant JL. Impact of folate and homocysteine metabolism on human reproductive health. Hum Reprod Update. 2007;13:225-38.

15. Mattson MP. Methylation and acetylation in nervous system development and neurodegenerative disorders. Ageing Res Rev. 2003;2:329-42.

16. Soubry A, Schildkraut JM, Murtha A et al. Paternal obesity is associated with IGF2 hypomethylation in newborns: results from a Newborn Epigenetics Study (NEST) cohort. BMC Med. 2013;11:29.

17. Parker S, Yazdy MM, Tinker SC, Mitchell AA, Werler MM. The impact of folic acid intake on the association among diabetes mellitus, obesity, and spina bifida. Am J Obstet Gynecol. 2013. doi:10.1016/j.ajog.2013.05.047i.

18. Li CCY, Young PE, Maloney CA, et al. Maternal obesity and diabetes induces latent metabolic defects and widespread epigenetic changes in isogenic mice. Epigenetics. 2013;1:8.

19. Martínez JA, Cordero P, Campión J, Milagro FI. Interplay of early-life nutritional programming on obesity, inflammation and epigenetic outcomes. Proc Nutr Soc. 2012;71:276-83.

20. Hollingsworth JW, Maruoka S, Boon K et al. In utero supplementation with methyl donors enhances allergic airway disease in mice. J Clin Invest. 2008;118:3462-9.

21. Stevens VL, McCullough ML, Pavluck AL, et al. Association of polymorphisms in one-carbon metabolism genes and postmenopausal breast cancer incidence. Cancer Epidemiol Biomarkers Prev. 2007;16:1140-7.

22. Richman EL, Kenfield SA, Stampfer MJ, et al. Choline intake and risk of lethal prostate cancer: incidence and survival. Am J Clin Nutr. 2012;96:855-63.

23. Xu X, Gammon MD, Zhang Y, et al. BRCA1 promoter methylation is associated with increased mortality among women with breast cancer. Breast Cancer Res Treat. 2009;115:397-404.

24. Lee JE, Giovannucci E, Fuchs CS, et al. Choline and betaine intake and the risk of colorectal cancer in men. Cancer Epidemiol Biomarkers Prev. 2010;19:884-7.

25. Ding Y, Li J, Liu S et al. DNA hypomethylation of inflammationassociated genes in adipose tissue of female mice after multigenerational high fat diet feeding. Int J Obes. 2013;1-7. doi:10. 1038/ijo.2013.98.

26. Reik W, Dean W, Walter J. Epigenetic reprogramming in mammalian development. Science. 2001;293:1089-93.

27. Tian X, Diaz FJ. Acute dietary zinc deficiency before conception compromises oocyte epigenetic programming and disrupts embryonic development. Dev Biol. 2013;376:51-61.

28. Wallwork JC, Duerre JA. Effect of zinc deficiency on methionine metabolism, methylation reactions and protein synthesis in isolated perfused rat liver. J Nutr. 1985;115:252-62.

29. Niculescu MD. Dietary choline deficiency alters global and genespecific DNA methylation in the developing hippocampus of mouse fetal brains. FASEB J. 2006;20:43-9. 
30. Albright CD, Friedrich CB, Brown EC, Mar MH, Zeisel SH. Maternal dietary choline availability alters mitosis, apoptosis and the localization of TOAD-64 protein in the developing fetal rat septum. Brain Res Dev Brain Res. 1999;115:123-9.

31. Albright CD, Mar MH, Friedrich CB, Brown EC, Zeisel SH. Maternal choline availability alters the localization of p15Ink4B and p27Kip1 cyclin-dependent kinase inhibitors in the developing fetal rat brain hippocampus. Dev Neurosci. 2001;23:100-6.

32. Davison JM, Mellott TJ, Kovacheva VP, Blusztajn JK. Gestational choline supply regulates methylation of histone h3, expression of histone methyltransferases G9a (Kmtlc) and Suv39h1 (Kmt1a), and DNA methylation of their genes in rat fetal liver and brain. J Biol Chem. 2008;284:1982-9.

33. - Niculescu MD, Lupu DS, Craciunescu CN. Perinatal manipulation of $\alpha$-linolenic acid intake induces epigenetic changes in maternal and offspring livers. FASEB J. 2013;27(1):350-8. Epigenetic effects induced by nutrient intake during preconception and/or gestation may be rescued by postnatal dietary changes.

34. Fox JT, Stover PJ. Folate-mediated one-carbon metabolism. Vitam Horm. 2008;79:1-44.

35. Feng $\mathrm{Y}$, Zhao L-Z, Hong L, et al. Alteration in methylation pattern of GATA-4 promoter region in vitamin A-deficient offspring's heart. J Nutr Biochem. 2013;24(7):1373-80.

36. Cong R, Jia Y, Li R, et al. Maternal low-protein diet causes epigenetic deregulation of HMGCR and CYP7 $\alpha 1$ in the liver of weaning piglets. J Nutr Biochem. 2012;23:1647-54.

37. Altmann SS, Murani EE, Schwerin MM, et al. Maternal dietary protein restriction and excess affects offspring gene expression and methylation of non-SMC subunits of condensin I in liver and skeletal muscle. Epigenetics. 2012;7:239-52.

38. - Pereira FF, Barbáchano AA, Singh PKP et al. Vitamin D has wide regulatory effects on histone demethylase genes. Cell Cycle. 2012;11:1081-9. Vitamin D regulates expression of histone demethylase genes in a complex regulatory feed back loop that suggests a greater involvement of active vitamin $D$ $[1,25(\mathrm{OH}) 2 \mathrm{D} 3]$ in regulating expression genome-wide.

39. Pereira F, Barbáchano A, Silva J, et al. KDM6B/JMJD3 histone demethylase is induced by vitamin $\mathrm{D}$ and modulates its effects in colon cancer cells. Hum Mol Genet. 2011;20:4655-65.

40. La Merrill MM, Torres-Sánchez LL, Ruiz-Ramos RR, et al. The association between first trimester micronutrient intake, MTHFR genotypes, and global DNA methylation in pregnant women. J Matern Fetal Neonatal Med. 2012;25:133-7.

41. McKay JA, Groom A, Potter C, et al. Genetic and non-genetic influences during pregnancy on infant global and site specific DNA Methylation: role for folate gene variants and vitamin B12. PLoS One. 2012;30(7):e33290.

42. Corbin KD, Abdelmalek MF, Spencer MD, et al. Genetic signatures in choline and 1-carbon metabolism are associated with the severity of hepatic steatosis. FASEB J. 2013;27:1674-89.

43. Niclot S. Implication of the folate-methionine metabolism pathways in susceptibility to follicular lymphomas. Blood. 2006;108:278-85.

44. Ulrich CM. Polymorphisms in the reduced folate carrier, thymidylate synthase, or methionine synthase and risk of colon cancer. Cancer Epidemiol Biomarkers Prev. 2005;14:2509-16.

45. Pogribny IP, Shpyleva SI, Muskhelishvili L, et al. Role of DNA damage and alterations in cytosine DNA methylation in rat liver carcinogenesis induced by a methyl-deficient diet. Mutat Res. 2009;669:7-8.

46. - Langie SAS, Achterfeldt S, Gorniak JP et al. Maternal folate depletion and high-fat feeding from weaning affects DNA methylation and DNA repair in brain of adult offspring. FASEB J. 2013. Maternal diet influences offspring susceptibility to postnatal dietary insult and can result in DNA damage that is maintained through adulthood. DNA damage was linked to decrease in DNA repair mechanisms present in both somatic and germ cells, introducing the possibility of germline transmission of such defects.

47. Hajkova P, Jeffries SJ, Lee C, Miller N, Jackson SP, Surani MA. Genome-wide reprogramming in the mouse germ line entails the base excision repair pathway. Science. 2010;329:78-82.

48. Kulkarni A, Dangat K, Kale A et al. Effects of altered maternal folic acid, vitamin $\mathrm{b}(12)$ and docosahexaenoic acid on placental global DNA methylation patterns in Wistar rats. PLoS One. 2011;6:e17706-6.

49. - Cordero PP, Gomez-Uriz AMA, Campion JJ, Milagro FIF, Martinez JAJ. Dietary supplementation with methyl donors reduces fatty liver and modifies the fatty acid synthase DNA methylation profile in rats fed an obesogenic diet. Genes Nutr. 2013;8:105-13. Links epigenetic effects of methyl donor nutrients to fatty acid metabolism pathway.

50. McNeil CJ, Beattie JH, Gordon M-J, Pirie LP, Duthie SJ. Differential effects of nutritional folic acid deficiency and moderate hyperhomocysteinemia on aortic plaque formation and genomewide DNA methylation in vascular tissue from ApoE-/- mice. Clin Epigenetics. 2011;2:361-8.

51. Carlin J, George R, Reyes TM. Methyl donor supplementation blocks the adverse effects of maternal high fat diet on offspring physiology. PLoS One. 2013;8:e63549-50.

52. Zeisel SHS. Gene response elements, genetic polymorphisms and epigenetics influence the human dietary requirement for choline. IUBMB Life. 2007;59:380-7.

53. Mikael LG, Pancer J, Jiang $X$ et al. Low dietary folate and methylenetetrahydrofolate reductase deficiency may lead to pregnancy complications through modulation of ApoAI and IFN$\gamma$ in spleen and placenta, and through reduction of methylation potential. Mol Nutr Food Res. 2012;57:661-70.

54. Ortega-Azorín CC, Sorlí JVJ, Asensio EME, et al. Associations of the FTO rs9939609 and the MC4R rs17782313 polymorphisms with type 2 diabetes are modulated by diet, being higher when adherence to the Mediterranean diet pattern is low. Cardiovasc Diabetol. 2012;11:137-8.

55. Pogribny IP, Kutanzi K, Melnyk S, et al. Strain-dependent dysregulation of one-carbon metabolism in male mice is associated with choline- and folate-deficient diet-induced liver injury. FASEB J. 2013;27:2233-43.

56. Tryndyak VV, de Conti AA, Kobets TT, et al. Interstrain differences in the severity of liver injury induced by a choline- and folatedeficient diet in mice are associated with dysregulation of genes involved in lipid metabolism. FASEB J. 2012;26:4592-602.

57. Dolinoy DC, Huang D, Jirtle RL. Maternal nutrient supplementation counteracts bisphenol A-induced DNA hypomethylation in early development. Proc Natl Acad Sci USA. 2007;104: 13056-61.

58. Rosenfeld CS, Sieli PT, Warzak DA, et al. Maternal exposure to bisphenol A and genistein has minimal effect on Avy/a offspring coat color but favors birth of agouti over nonagouti mice. 2013;110:537-42.

59. Xing LL, Xu YY, Xiao YY, et al. Embryotoxic and teratogenic effects of the combination of bisphenol $\mathrm{A}$ and genistein on in vitro cultured post implantation rat embryos. Toxicol Sci. 2010;115:577-88.

60. Gertz JJ, Reddy TET, Varley KEK, Garabedian MJM, Myers RMR. Genistein and bisphenol A exposure cause estrogen receptor 1 to bind thousands of sites in a cell type-specific manner. Genes Dev. 2012;22:2153-62.

61. - Tsang VV, Fry RCR, Niculescu MDM et al. The epigenetic effects of a high prenatal folate intake in male mouse fetuses exposed in utero to arsenic. Toxicol Appl Pharmacol. 2012;264:439-50. Aggregate effects of prenatal folate 
supplementation and arsenic exposure suggests that the risk of arsenic exposure must be taken into consideration when considering folate supplementation during pregnancy-a concept that may apply to other toxicants or dietary supplements.

62. Nohara KK, Baba TT, Murai HH, et al. Global DNA methylation in the mouse liver is affected by methyl deficiency and arsenic in a sex-dependent manner. Arch Toxicol. 2011;85:653-61.
63. Furrow RE, Christiansen FB, Feldman MW. Environment-sensitive epigenetics and the heritability of complex diseases. Genetics. 2011;189:1377-87.

64. Li MJ, Wang LY, Xia Z, Sham PC, Wang J. GWAS3D: detecting human regulatory variants by integrative analysis of genomewide associations, chromosome interactions and histone modifications. Nucleic Acids Res. 2013;41:W150-8. 